\title{
Organic cocrystals: the development of ferroelectric properties
}

\author{
Geetha Bolla $^{1}$, Huanli Dong ${ }^{1 *}$, Yonggang Zhen ${ }^{1 *}$, Zhaohui Wang ${ }^{1}$ and Wenping $\mathrm{Hu}^{1,2}$
}

\begin{abstract}
Organic cocrystals are crystalline, single-phase materials composed of two or more molecular and/or ionic compounds, generally, in a stoichiometric ratio. A feature of organic cocrystals is that special optoelectronic properties such as ferroelectricity are easy to realize in these materials. In this perspective, we systematically introduce the recent research advances in organic cocrystal ferroelectrics, and we study in depth the molecular structure and self-assembling behaviors of cocrystals for ferroelectric applications. Finally, combined with an understanding of recent progress and achievements in this field, we discuss the challenges and opportunities for ferroelectric materials based on organic cocrystals, as well as the promising applications of these materials.
\end{abstract}

Keywords: organic cocrystal, hydrogen bonding, charge transfer, ferroelectric

An organic cocrystal is a stoichiometric, multi-component molecular crystal wherein the different components are assembled by heteromolecular interactions (Fig. 1a), such as hydrogen bonds, halogen bonds, charge transfer (CT), and $\pi-\pi$ interactions [1]. In cocrystalline systems, the supramolecular synthon [2], a fundamental concept of crystal engineering, plays an important role. Synthons are reliable patterns of intermolecular interactions that can be used to generate supramolecular functional materials. The first cocrystal was reported by Wöhler [3] in 1844 during his studies on quinone. He mixed solutions of quinone (colorless) and hydroquinone (yellow) and found that a crystalline substance formed. Wöhler called this crystalline material green hydroquinone. Following this first publication, Ling and Baker reported several related cocrystals composed of halogenated quinones and green hydroquinone, also referred to as quinhydrone [4]. In
1958, the first crystal structure of a monoclinic quinhydrone crystal was reported [5]. The crystal structure showed that the quinone and hydroquinone molecules formed alternating zigzag chains connected by $\mathrm{O}-\mathrm{H} \cdots \mathrm{O}$ hydrogen bonds. Another crystal engineering strategy relies on the use of electronic donor (D) and acceptor (A) building blocks. This process has gained considerable interest because large molecules can be utilized and the resultant DA cocrystals are stable. Moreover, these types of cocrystal have the potential to be used for the fabrication of promising next generation materials with applications in innovative electronic and photonic devices [6]. CT interactions mainly refer to weak, non-covalent attractive forces that arise between electron-rich donors and electron-deficient acceptors, forming so-called D-A complexes. However, these CT cocrystals have been investigated to a lesser degree than cocrystals held together by hydrogen bonds.

The properties of cocrystals are not thoroughly investigated in terms of their technological applications, especially concerning optical nonlinearity, (semi)conductivity, ferroelectricity, and magnetism [7]. In the last decade, multifunctional organic materials have emerged as high-demand materials because they are versatile and have applications in the area of molecular optoelectronics. However, there has been limited success in developing optoelectronically active materials using traditional, single-component materials. However, the formation of supramolecular assemblies of materials into cocrystals through non-covalent interactions allows material scientists to tailor the properties of the final material by careful choice of multifunctional component molecules, yielding materials with unique physicochemical properties [8]. Now, having dis-

\footnotetext{
${ }^{1}$ Key laboratory of Organic Solids, Institute of Chemistry, Chinese Academy of Sciences, Beijing 100190, China

2 Tianjin Key Laboratory of Molecular Optoelectronic Sciences, Department of Chemistry, School of Science, Tianjin University \& Collaborative Innovation Center of Chemical Science and Engineering (Tianjin), Tianjin 300072, China

* Corresponding authors (emails: dhl522@iccas.ac.cn (Dong H); zhenyg@iccas.ac.cn (Zhen Y))
} 
a

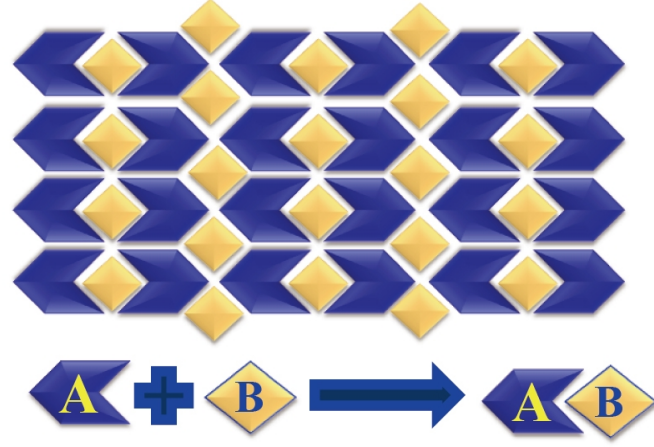

$A=A P I, B=$ coformer, solvent, salt former A.B=cocrystal, solvate, molecular salt

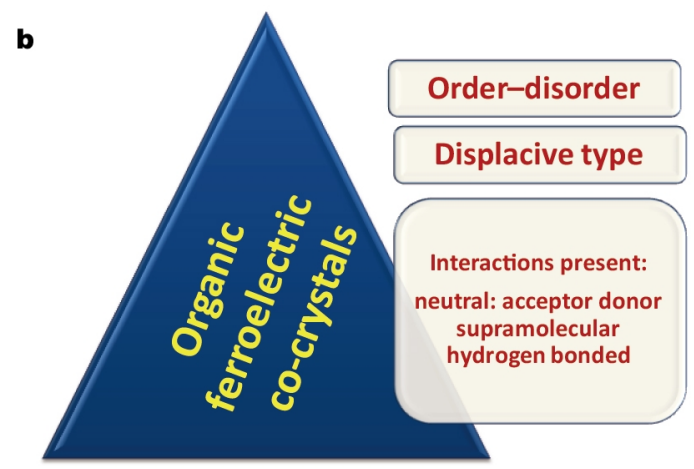

Figure 1 (a) General schematic representation of cocrystals. (b) Types of ferroelectric systems and the interactions in these crystal structures.

cussed the background to cocrystalline materials, we would like to explore their applications; in particular, we will focus on organic ferroelectrics and studies into the correlation between their two dimensional (2D) surface arrangements and properties [9].

Ferroelectrics are polar (pyroelectric) crystals that show spontaneous electric polarization. They have electroactive properties, allowing the storage and switching of polarity (ferroelectricity), sensing of temperature changes (pyroelectricity), interchange of electric and mechanical functions (piezoelectricity), and manipulation of light (through optical nonlinearities and the electro-optical effect) [10]. Ferroelectric materials are mostly inorganic or organic-inorganic hybrid compounds, for example, barium titanate $\left(\mathrm{BaTiO}_{3}\right)(\mathrm{BTO})$, sodium nitrite $\left(\mathrm{NaNO}_{2}\right)$, potassium dihydrogen phosphate $\left(\mathrm{KH}_{2} \mathrm{PO}_{4}, \mathrm{KDP}\right)$, and triglycine sulfate (TGS) [11]. Historically, ferroelectrics can be traced back to 1655, when Rochelle salt was first isolated by Elie Seignette, but pyroelectricity has been recognized since ancient times. However, the piezoelectricity of Rochelle salt was established in 1880, by Curie's brother, and it took a further 40 years, in 1920 , until the term 'ferroelectric' was coined by Joseph Valasek by analogy with ferromagnetism. For some time, Rochelle salt was the only known ferroelectric material; however, in 1935, Busch and Scherer found KDP to be ferroelectric. A further breakthrough came in the 1940s, during the Second World War, when BTO, which has a simple perovskite structure, was prepared, followed by von Hippel's demonstration of its ferroelectricity in 1945 [12]. Following this period of discovery, piezoelectric transducers were developed, and many new ferroelectric perovskite oxides were synthesized. Among these new materials, the most notable is lead zirconate titanate (PZT). Today, PZT remains the most widely used ferroelectric material [13]. In summary, inorganic ferroelectrics have been well explored, and for organic ferroelectrics, the specifics are well established. Currently, for organic ferroelectrics, attention is given to the use of multicomponent materials that exhibit ferroelectricity and polarization at temperatures at and above room temperature.

Conventional organic ferroelectrics [14] are of the order-disorder type (Figs $1 \mathrm{~b}$ and 2), as will be discussed later. The most interesting feature of ferroelectric materials is their spontaneous polarization, which can be reversed by inverting the external electric field. During polarization reversal in a coercive field, the electric displacement (D) as a function of the field (E) exhibits a hysteresis (a D-E loop), and ferroelectric materials undergo a paraelectric-to-ferroelectric phase transition. Furthermore, dielectric susceptibility usually obeys the Curie-Weiss law at high temperatures, i.e., temperatures above the Curie temperature $\left(T_{\mathrm{c}}\right)$. Normally, ferroelectric phase transitions are categorized using the following scheme. 1. Order-disorder: in this scheme, the ordering of the asymmetric molecules or ions carrying permanent dipoles generates a spontaneous electric polarization. Sodium nitrite is a well-known example, in which the bent $\mathrm{NO}_{2}{ }^{2-}$ ion bears the electric dipole (Fig. 2a). 2. Displacive type: in the displacive mechanism, the relative displacements between the different charges of the ions yield macroscopic polarization in the crystal, an example being perovskite ferroelectrics such as BTO (Fig. 2). The ferroelectric phenomenon can be utilized for a diverse range of technological applications such as non-volatile memory elements (ferroelectric random access memory (FeRAM)) or ferroelectric field-effect transistors (FeFET), and high-capacity condensers and capacitors. Furthermore, ferroelectric crystals exhibit unusually large electromechanical couplings, i.e., the coupling between mechanical strain and electric polarization. The large electrostriction and piezoelectric effect can be utilized 
a

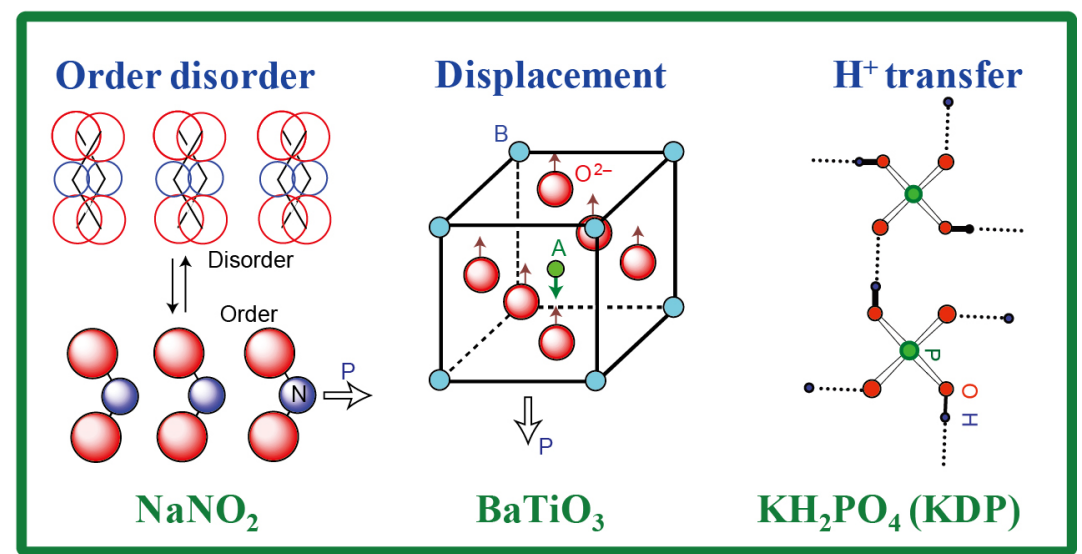

b

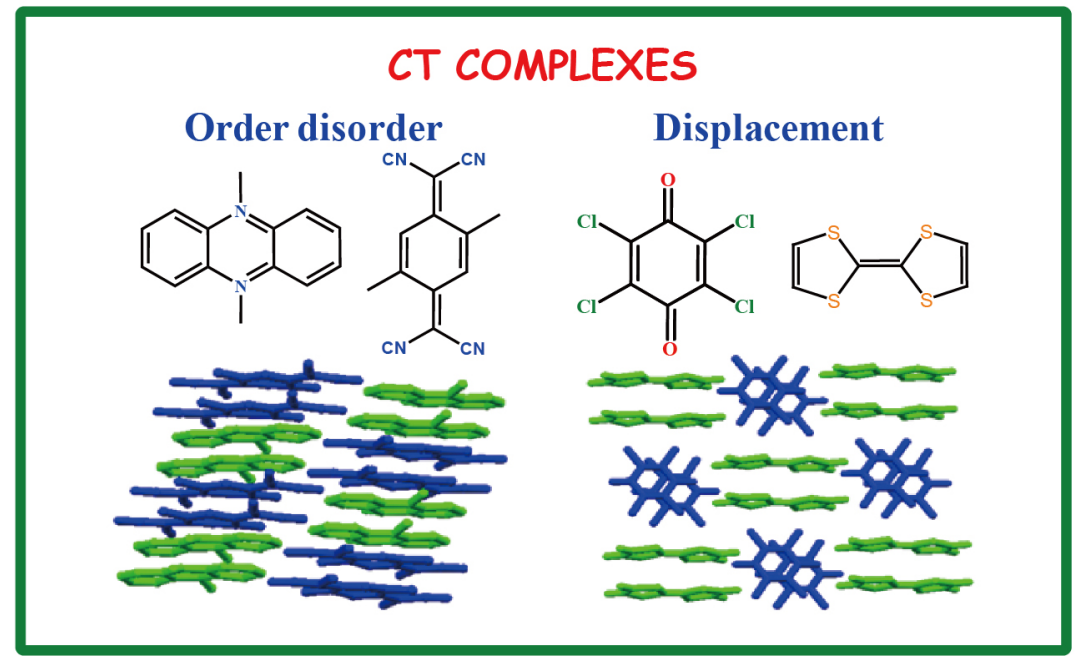

Figure 2 (a) Conventional classifications of ferroelectric materials in inorganic chemistry. The origin of the dipole moment, $p$, is shown by open arrows. (b) Classical examples of organic ferroelectrics CT complexes with their classification and 2D packing. Reprinted with permission from Ref. [9], Copyright 2008, Nature Publishing Group.

in actuators, transducers, ultrasonic motors, piezoelectric elements, and second-order optical nonlinearity for second harmonic generation (SHG) activity along with the linear electro-optic effect. Moreover, there are many challenges, and these are broadly interdisciplinary, for example, the development of frequency multipliers and light modulators and photonic devices, which are currently of great interest and also could be fabricated using crystal engineering methods.

\section{Ferroelectric organic cocrystals}

Recently, the CT strategy for the formation of cocrystals has been applied to form a complex of tetrathiafulvalene (TTF) and p-chloranil $\left(\mathrm{QCl}_{4}\right)$, resulting in $\pi$-stacked columns of alternating D and A ("mixed stack") molecules in one dimension. This one-dimensional stacking is vital in the formation of ferroelectric properties (Figs $2 b$ and 3 )
[15]. The most important organic molecules and binary systems, along with their properties, are summarized in Table 1. Occasionally, strong electron-lattice interactions (Peierls instability) trigger molecular displacements. These displacements lead to D-A dimerization, forming electric dipoles along the molecular stack. In addition, the flat $\mathrm{D}$ and $\mathrm{A}$ molecules are flexible enough to bend, and the spontaneous bending deformation breaks the symmetry, generating highly polar D-A chains. The interactions and the arrangement of the molecules in the crystal lattice lead to either a ferroelectric or antiferroelectric state, depending on the three dimensional (3D) arrangement of the polar chains. The ferroelectric and antiferroelectric ordering styles are both shown by TTF- $\mathrm{QCl}_{4}$ on phase transition, and the dielectric properties have been closely controlled in various ways depending on the chemical and physical modifications of the crystals. However, these CT com- 

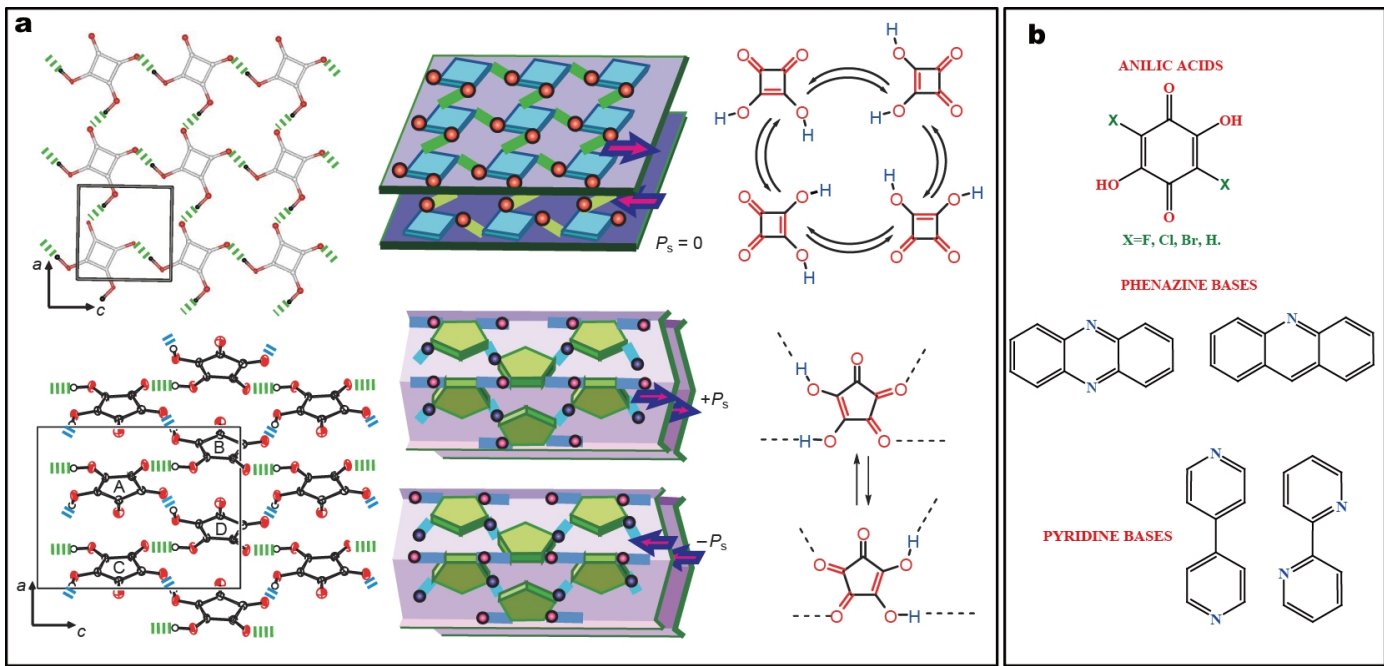

Figure 3 (a) Structural comparisons between an antiferroelectric squaric acid (top) and ferroelectric croconic acid (bottom). Reprinted with permission from Ref. [20], Copyright 2010, Nature Publishing Group. (b) Chemical structures of generally used anilic acids and base molecules of phenazine, bipyridines reported in literatures for ferroelectric organic adducts.

Table 1 Properties of purely organic ferroelectrics compared with those of selected typical ferroelectrics of small single compounds and organic complexes compounds (modified from Ref. [9])

\begin{tabular}{|c|c|c|c|c|c|}
\hline & \multicolumn{2}{|c|}{ Transition temperature $(\mathrm{K})$} & \multicolumn{2}{|c|}{ Dielectric constant } & \multirow{2}{*}{ Ps $\left(\mu \mathrm{C} \mathrm{cm}{ }^{-2}\right)$; temperature } \\
\hline & $T_{\mathrm{c}}$ & $T_{\mathrm{c}}^{\mathrm{D}}$ & $\kappa_{\mathrm{RT}}$ & $\kappa_{\max }$ & \\
\hline \multicolumn{6}{|c|}{ Single-component organic molecules } \\
\hline Thiourea & 169 & 185 & 30 & 104 & $3.2 ; 120 \mathrm{~K}$ \\
\hline TEMPO & 287 & 288 & 10 & 16 & 0.5 \\
\hline CDA & 397 & & & 25 & \\
\hline TCAA & 355 & & 4.5 & 6.5 & $0.2 ; \mathrm{RT}$ \\
\hline Benzil & 78 & 84 & 88 & 2.7 & $3.6 \times 10^{-3} ; 70 \mathrm{~K}$ \\
\hline DNP & 46 & & 4.0 & 22 & \\
\hline TCHM & 24 & 104 & 9.6 & 100 & $0.24 ; 10 \mathrm{~K}$ \\
\hline \multicolumn{6}{|c|}{ CT complexes } \\
\hline TTF-CA & 81 & 84 & 40 & 500 & \\
\hline TTF-BA & 50 & & & 20 & \\
\hline \multicolumn{6}{|c|}{ H-bonded supramolecules } \\
\hline $\mathrm{Phz}-\mathrm{H}_{2} \mathrm{Ca}$ & 253 & 304 & 110 & $3 \times 10$ & $1.8 ; 160 \mathrm{~K}$ \\
\hline $\mathrm{Phz}-\mathrm{H}_{2} \mathrm{ba}$ & 138 & 204 & 30 & $1.7 \times 10^{3}$ & $0.8 ; 105 \mathrm{~K}$ \\
\hline
\end{tabular}

CDA = cyclohexan-1,1'-diacetic acid; DNP = 1,6-bis (2,4-dinitrophenoxy)-2,4-hexadiyne; TCAA = trichloroacetamide; TCHM = tricyclohexylmethanol; TEMPO = 2,2,6,6-tetramethyl-1-piperidinyloxy(tanane); $T_{\mathrm{c}}{ }^{\mathrm{D}}=$ transition point for deuterated compound, $\kappa_{\mathrm{RT}}$, dielectric constant at room temperature; $\kappa_{\max }$, maximum dielectric constant at $T_{\mathfrak{c}} ; P_{\mathrm{s}}=$ spontaneous polarization.

plexes exhibit a relatively narrow charge gap and, hence, are bad insulators. In addition, large dielectric losses degrade the spontaneous polarization, preventing various ferroelectric applications near or above room temperature.

Hydrogen-bonded organic compounds are another type of cocrystalline material. Concerning ferroelectricity, these materials can behave in a similar way to KDP. Ferro- electricity and the related properties of hydrogen-bonded systems have been studied in many inorganic molecular salt systems [16-17], and KDP is a well-known ferroelectric material. $\mathrm{PO}_{4}{ }^{3-}$ ions are connected in a $3 \mathrm{D}$ interpenetrating network of homonuclear $\mathrm{O}-\mathrm{H} \cdots \mathrm{O}$ hydrogen bonds with short $\mathrm{O}$... O separations (2.50 $\mathrm{A})$. A phase transition is triggered by the collective ordering of protons, and the 
magnitude of spontaneous polarization of the system arises mainly from the displacive motion of highly polarizable $\mathrm{PO}_{4}{ }^{3-}$ ions. Furthermore, these protons can be replaced by deuterons, leading to a dramatic increase in crystallizing temperature $\left(T_{\mathrm{c}}\right)$. Therefore, both hydrogen bonding and proton dynamics are very important in ferroelectric materials. There are several prototypical materials for this type of behaviors, for example, squaric acid. The square planar $\mathrm{C}_{4} \mathrm{O}_{4} \mathrm{H}_{2}$ molecules of squaric acid form strong hydrogen bonds with neighboring squaric acid molecules; again, these have a short $\mathrm{O} \cdots \mathrm{O}$ distances $(2.55 \AA)$, forming a $2 \mathrm{D}$ network via intermolecular $\mathrm{O}-\mathrm{H}$... O hydrogen bonds. In this crystal, a phase transition at $T=373 \mathrm{~K}$ is a collective order-disorder of the protons, and this crystal, like KDP, has a significant $\mathrm{H} / \mathrm{D}$ isotope effect. This constraint, the so-called "ice rule", characterizes the correlated motion of protons. True organic ferroelectric analogs of KDP are desirable, in particular, multi-component, crystal-engineered systems. Displacive-type ferroelectricity in neutral cocrystals has been well discussed, as has proton transfer in acid-base multi-component molecules mediated by intermolecular hydrogen bonds. Indeed, initially, a supramolecular system is a system of hydrogen-bonded nonpolar molecules, whereas subsequent proton transfer yields an ionic supramolecular system. These types of ferroelectric compounds mainly include anilic acids as the proton donors, $\mathrm{D}$, and pyridine bases as the acceptors, $\mathrm{A}$. For example, anilic acid and bipyridine form ionic DA adducts on proton transfer of an anilic acid proton to bipyridine. Furthermore, these cocrystals have a variety of supramolecular arrangements, as shown in Fig. $3 b$.

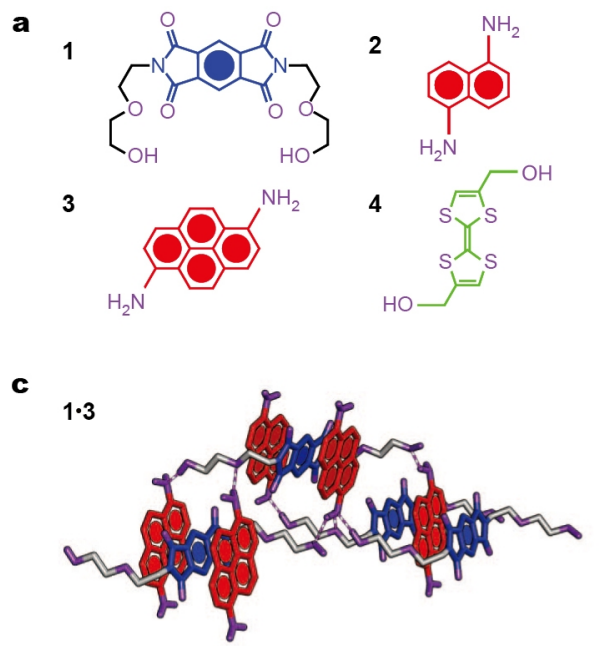

Subsequently, the drawbacks of this pioneering work were resolved by replacing CT interactions with hydrogen bonding cocrystals, i.e., the lock arm supramolecular ordering (LASO) method, which has succeeded in producing room temperature ferroelectric materials (Fig. 4), a molecular design that allows donor and acceptor molecules to self-assemble into charge-transfer ferroelectric networks at ambient temperature [18-19]. New structures arising from this technique challenge the long-standing notion that donor-acceptor mixed-stack materials cannot exhibit a ferroelectric $T_{\mathrm{c}}$ greater than room temperature. The demonstration of ferroelectric properties in an organic network affords us opportunities to produce these systems in new forms, such as electrically addressable hydrogels, ferroelectric catalysts, and charge-transfer-based sensitizers for photovoltaics. The combination of donor-acceptor interactions with hydrogen-bonded networks above room temperature [20] offers a promising supramolecular platform to design novel organic electronic structures.

\section{D organic cocrystals with potential ferroelectrics}

Recently, 2D H-bonded organic cocrystals of croconic acid (CA) and 3-hydroxyphenalenone (3-HPLN) have been prepared by self-assembly on an $\mathrm{Au}(111)$ substrate, as shown by scanning tunneling microscopy images (STM) in Fig. 5 [21], and these 2D materials show ferroelectric at room temperature. The CA/3-HPLN system forms cocrystals with a variety of stoichiometries. One of the observed structural building blocks consists of two CA and two 3-HPLN molecules. Studies have shown that CA and 3-HPLN can be cocrystallized as 2D crystals in a solvent-
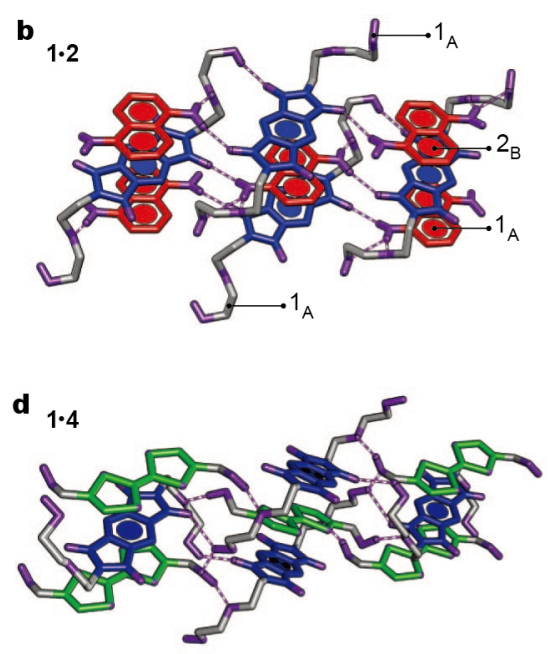

Figure 4 Crystal structures of LASO complexes and structural formula of the electron donor and acceptor molecules used. Reprinted with permission from Ref. [14], Copyright 2012, Nature Publishing Group. 

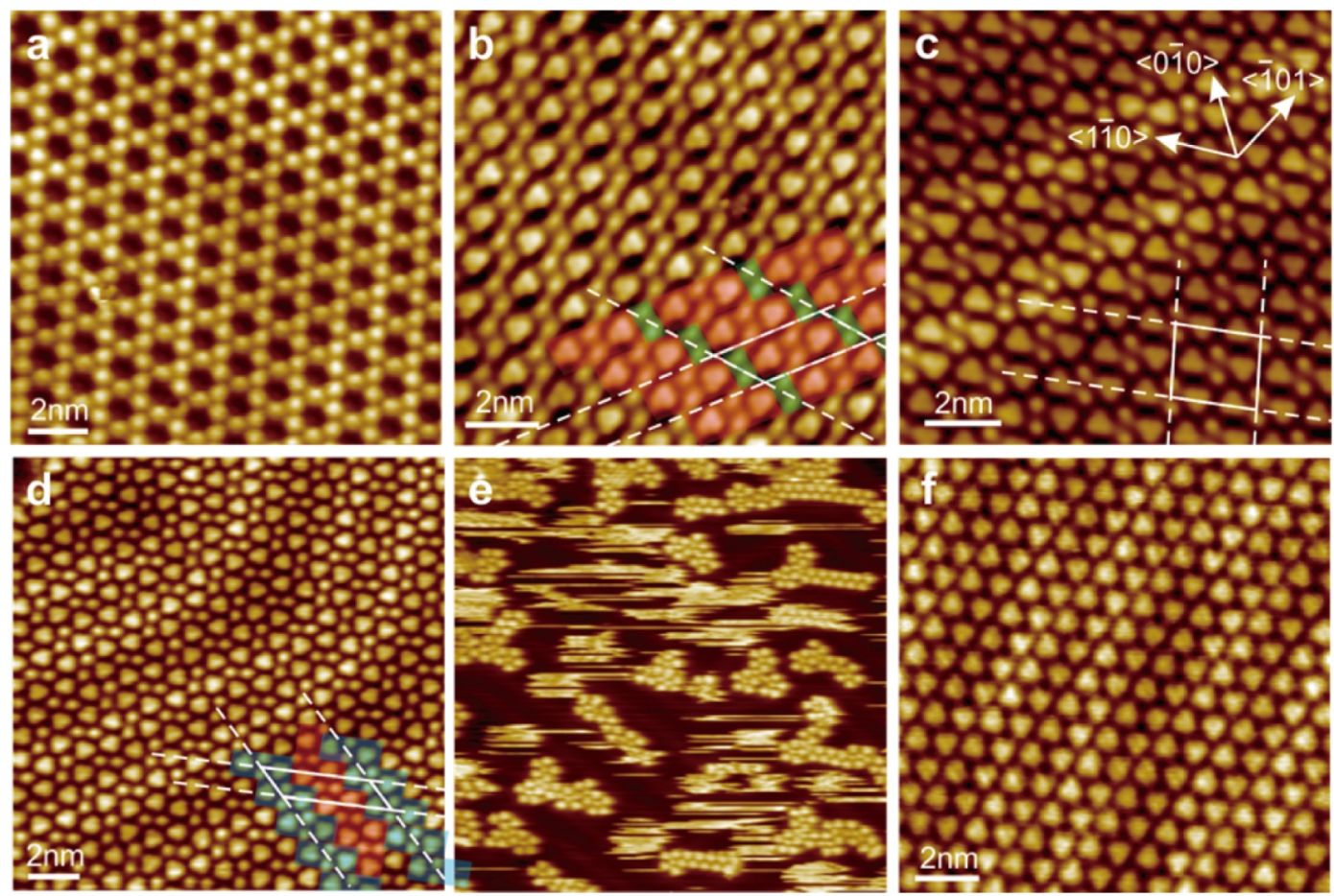

Figure 5 STM images of the self-assembled 2D molecular structures on an Au (111) substrate. (a) CA; (b) CA/3-HPLN $=2: 1$, where $(\mathrm{CA})_{2}(3-\mathrm{HPLN})_{2}$ tetramers are shaded red and $(\mathrm{CA})_{2}$ dimers are shaded green; $(c, d)$ two network architectures of CA/3-HPLN $=1: 1$ that were found to coexist (packing polymorphism), (CA) 2 (3-HPLN) 2 ; (e) CA/3-HPLN at approximately 1:5; and (f) 3-HPLN. Reprinted with permission from Ref. [20], Copyright 2010, Nature Publishing Group.

free approach when confined by a substrate. The importance of this study lies not only in the formation of a cocrystalline material but the solvent-free approach and surfacesupported cocrystallization. Thus, this method could become a quick and versatile test to identify miscible cocrystal constituents and inform solution-based cocrystallization, furthering the discovery of new materials and enabling the rational design of organic ferroelectric cocrystals. We hope that this work will encourage the search for cocrystalline organic $\mathrm{H}$-bonded systems, particularly those that are prone to network formation, which can prevent crystallization in centrosymmetric space groups, thus allowing ferroelectric phenomena in $2 \mathrm{D}$ materials to be generated.

\section{Applications for future studies of organic ferroelectrics}

Ferroelectric materials have important technological applications because of their superior piezoelectric properties. In the last a few years, a number of new molecular ferroelectric materials have been developed, and these are soft, flexible, lightweight, environmentally friendly, and biocompatible; in addition, they can be easily processed at relatively low temperatures and printed onto soft substrates. These materials are promising and have a broad range of applications, including sensing, actuation, energy harvesting, data storage, and memory devices (such as FeRAM and FeFETs). Another distinct advantage of molecular ferroelectrics is that they can be directly printed, as demonstrated in an all-printed ferroelectric active-matrix sensor network and in energy harvesting devices that convert mechanical vibrations into electricity. With this background, we expect that newly developed molecular ferroelectrics based on crystal engineering design strategies using cocrystals design will enter the field soon, thus enabling many more applications. As mentioned above, organic cocrystals are a popular research topic in material science in the form of CT complexes. In addition, photonic and optoelectronic compounds are becoming increasingly popular. Therefore, the synthesis of a wide variety of organic cocrystals is highly valuable, and applications of the aspects of crystal engineering to organic cocrystals will allow the design of highly desirable material properties. The application of molecular cocrystals and understanding their crystal structures and interactions will significantly advance the preparation of improved functional materials. The design of the hydrogen-bonded supramolecular materials that are ferroelectric at and above room temperature, have excellent charge transport properties, have diverse applications, and 
show photonic behavior will lead to further developments in molecular electronics in the next couple of decades.

Received 11 April 2016; accepted 6 June 2016; published online 14 July 2016

1 Aitipamula S, Banerjee R, Bansal AK, et al. Polymorphs, salts, and cocrystals: what's in a name?. Crystal Growth Design, 2012, 12: 2147-2152

2 Desiraju GR. Supramolecular synthons in crystal engineering-a new organic synthesis. Angew Chem Int Ed, 1995, 34: 2311-2327

3 Wöhler F. "Untersuchungen Über des Chinons". Annalen Chem Pharm, 1844, 51: 145-163

4 Ling AR, Baker JL. XCVI. halogen derivatives of quinone. Part III. Derivatives of quinhydrone. J Chem Soc Trans, 1893, 63: 1314-1327

5 Matsuda H, Osaki K, Nitta I. Crystal structure of quinhydrone, C12H10O4. Bull Chem Soc Jpn, 1958, 31: 611-620

6 Zhu W, Zheng R, Zhen Y, et al. Rational design of charge-transfer interactions in halogen-bonded co-crystals toward versatile solidstate optoelectronics. J Am Chem Soc, 2015, 137: 11038-11046

7 Li J, Liu Y, Zhang Y, et al. Molecular ferroelectrics: where electronics meet biology. Phys Chem Chem Phys, 2013, 15: 20786

8 Zhu W, Zheng R, Fu X, et al. Revealing the charge-transfer interactions in self-assembled organic cocrystals: two-dimensional photonic applications. Angew Chem Int Ed, 2015, 54: 6785-6789

9 Horiuchi S, Tokura Y. Organic ferroelectrics. Nat Mater, 2008, 7: 357-366

10 Lines ME, Glass AM. Principles and Applications of Ferroelectrics and Related Materials. New York: Oxford University Press, 1977

11 K. Uchino. Ferroelectric Devices. New York: CRC Press, 2000

12 Von hippel A, Breckenridge RG, Chesley FG, et al. High dielectric constant ceramics. Ind Eng Chem, 1946, 38: 1097-1109

13 Shirane G, Takeda A. Phase transitions in solid solutions of $\mathrm{PbZrO}_{3}$ and $\mathrm{PbTiO}_{3}(\mathrm{I})$ small concentrations of $\mathrm{PbTiO}_{3}$. J Phys Soc Jpn, 1952, 7: 5-11
14 Tayi AS, Shveyd AK, Sue ACH, et al. Room-temperature ferroelectricity in supramolecular networks of charge-transfer complexes. Nature, 2012, 488: 485-489

15 Torrance JB, Girlando A, Mayerle JJ, et al. Anomalous nature of neutral-to-ionic phase transition in tetrathiafulvalene-chloranil. Phys Rev Lett, 1981, 47: 1747-1750

16 Katrusiak A, Szafrański M. Ferroelectricity in NH $\cdots \mathrm{N}$ hydrogen bonded crystals. Phys Rev Lett, 1999, 82: 576-579

17 Mochida T, Izuoka A, Sugawara T, et al. Organic hydrogen-bonded dielectrics: quantum paraelectricity based on tautomerization of 9-hydroxyphenalenone derivatives. J Chem Phys, 1994, 101: 7971-7973

18 Tayi AS, Kaeser A, Matsumoto M, et al. Supramolecular ferroelectrics. Nat Chem, 2015, 7: 281-294

19 Blackburn AK, Sue ACH, Shveyd AK, et al. Lock-arm supramolecular ordering: a molecular construction set for cocrystallizing organic charge transfer complexes. J Am Chem Soc, 2014, 136: 17224-17235

20 Horiuchi S, Tokunaga Y, Giovannetti G, et al. Above-room-temperature ferroelectricity in a single-component molecular crystal. Nature, 2010, 463: 789-792

21 Kunkel DA, Hooper J, Bradley B, et al. 2D cocrystallization from H-bonded organic ferroelectrics. J Phys Chem Lett, 2016, 7: 435-440

Acknowledgments This work was supported by the National Natural Science Foundation of China (91222203, 91233205, 51222306, 91027043 and 91433115), the Ministry of Science and Technology of China (2013CB933403, 2013CB933500 and 2014CB643600), the Strategic Priority Research Program of the Chinese Academy of Sciences (XDB12030300), and the Chinese Academy of Sciences.

Author contributions Bolla G wrote the paper and Dong H, Zhen Y, Wang $\mathrm{Z}$, and $\mathrm{Hu} \mathrm{W}$ gave discussion in depth on the content of this article.

Conflict of interest The authors declare that they have no conflicts of interest. 

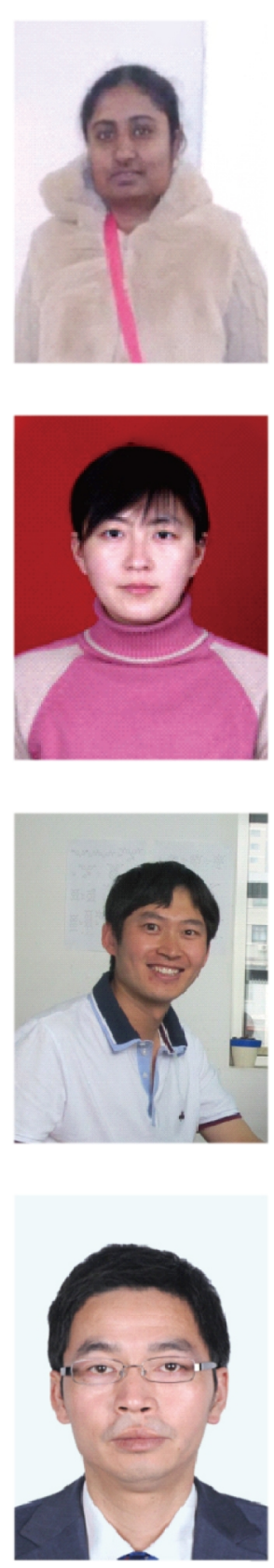

Geetha Bolla obtained her PhD degree at the University of Hyderabad, India and she is now a postdoctoral researcher in the Laboratory of Organic Solids, Institute of Chemistry, Chinese Academy of Sciences (ICCAS). Her research interests are design, synthesis, and applications of organic cocrystals.
Huanli Dong is an associate professor of the ICCAS. She grew up in Shandong province, China. She received her $\mathrm{PhD}$ degree from the ICCAS in 2009, after getting MSc degree at Fujian Institute of Research on the Structure of Material, CAS in 2006. She is presently focusing on the self-assembling of molecular materials and the applications of molecular materials in optoelectronic devices. Presently, she has more than 110 peer review publications with citation more than 2900 times (H-index=24).

Yonggang Zhen is an associate professor of the ICCAS. He grew up in Shanxi province, China. He received his PhD degree from the ICCAS in 2011. He is presently focusing on the design and synthesis of novel organic semiconductors.
Zhaohui Wang is a professor of the ICCAS. He received his $\mathrm{PhD}$ from East China University of Science and Technology in 1999. He then joined Max Planck Institute for Polymer Research as a postdoctoral research fellow. In 2005, he joined the ICCAS and was promoted to full professor. His research focuses on the design and synthesis of novel organic semiconductors.

\section{有机共晶铁电材料的研究进展}

Geetha Bolla ${ }^{1}$, 董焕丽 ${ }^{1 *}$, 甄永刚 ${ }^{*}$, 王朝晖 ${ }^{1}$, 胡文平 ${ }^{1,2}$

摘要 “有机共晶” 作为一种由两种或者两种以上分子按照一定比例形成的多组份体系在实现一些物理化学特性方面显示了独特的优势, 譬如铁电特性. 本文系统介绍了有机共晶铁电材料方面的研究进展, 深入分析了有机共晶在实现铁电性能方面的分子结构特征、共晶体 系组装策略、以及铁电性能. 最后, 结合对这些研究进展和结果的分析, 作者指出了利用有机共晶实现铁电特性研究领域中所存在的各种 挑战与机遇以及有机共晶铁电材料潜在的应用前景. 